\title{
Les entreprises et les temps de la globalisation
}

Gabriel Galvez-Behar (IRHIS-Université de Lille) - Philippe Lefebvre (CGS Mines ParisTech)

à paraître dans Entreprises et Histoire, 2019/1, article introductif au numéro spécial Globalisation et transformation des entreprises

Le terme de mondialisation apparaît dès le début du XX $\mathrm{X}^{\mathrm{e}}$ siècle et celui de globalisation, en anglais, se répand durant la Seconde Guerre mondiale à la faveur d'une mutation de l'imaginaire géographique $^{1}$. La publication en 1968 de l'ouvrage de Marshall McLuhan et de Quentin Fiore, War and Peace in the Global Village, marque un autre jalon important de cette dynamique ${ }^{2}$. L'article de Theodore Levitt, «The globalization of markets », publié en mai 1983 dans la Harvard Business Review semble marquer l'entrée du terme dans la communauté académique qui s'intéresse aux entreprises et symbolise sur ce plan l'émergence de la mondialisation actuelle ${ }^{3}$. Depuis le milieu des années 1980, les études sur la globalisation et la mondialisation n'ont cessé de se développer. Notre revue leur a consacré d'ailleurs un numéro en 2003 et la réunion annuelle de la Business History Conference de 2019 a porté également sur ce thème ${ }^{4}$. L'ampleur du thème, son renouvellement

1 René Dagorn, «Une brève histoire du mot "mondialisation" » in Michel Beaud et al., Mondialisation. Les mots et les choses, Paris, Karthala, 1999, p. 187-204 ; Olaf Bach, Die Erfindung der Globalisierung: Entstehung und Wandel eines zeitgeschichtlichen Grundbegriffs, Frankfurt, Campus Verlag, 2013 ; Vincent Capdepuy, «L'histoire d'un mot », Alternatives économiques, "Mondialisation et démondialisation », hors-série $\mathrm{n}^{\circ}$ 101, avril 2014, en ligne : https://www.alternatives-economiques.fr/lhistoire-dun/00061671 consulté le 29 avril 2019 ; Vincent Capdepuy, «L'entrée des États-Unis dans l'« âge global» : un tournant géohistorique?»,Monde(s), vol. 8, nº 2, 2015, p. 177-196.

2 Marshall McLuhan et Quentin Fiore, War and Peace in the Global Village. An inventory of some of the current spastic situations that could be eliminated by more feedforward, coordonné par Jerome Agel, NewYork, McGraw-Hill, 1968.

3 Theodore Levitt, « The Globalization of Markets », Harvard Business Review, vol. 61, n 3, mai-juin 1983, p. $92-102$.

4 Dominique Barjot (ed.), «Globalization - Mondialisation », Entreprises et histoire, n 32, 2003. Cette publication s'est faite dans le sillage d'autres numéros et notamment : "L'implantation internationale », Entreprises et histoire, $\mathrm{n}^{\circ}$ 8, juin 1995 ; le thème de la Business History Conference tenue en mars 2019 à Carthagène (Colombie) était : «Globalization and Deglobalization ; Shifts of Power and Wealth ». 
incessant (la mondialisation est un processus toujours à l'œuvre), l'importance des questions qu'il soulève - voilà autant d'éléments qui justifient l'intérêt constant pour ce thème et les tentatives de renouvellement d'éclairage à son sujet.

Pour sa part, ce numéro entend moins traiter de la globalisation en général que des manières de lire certaines transformations importantes des entreprises en rapport à la mondialisation (les mondialisations, plus exactement). Schématiquement, le parti pris est le suivant : examiner d'abord certaines des formes de mondialisation qui ont pesé sur la mondialisation des entreprises, cette mondialisation des entreprises étant alors vue comme une adaptation à ou une suite assez logique à d'autres formes de mondialisation (cf. les trois premiers articles de ce numéro); examiner ensuite comment, par leurs choix de découpage des activités, d'implantation géographique de ces sousactivités voire d'externalisation de ces activités, les entreprises ne se contentent pas de s'adapter à un phénomène qui les dépasse mais contribuent elles-même à construire ce qu'on appelle « mondialisation » (cf. les trois articles suivants de ce numéro); enfin, que les entreprises s'adaptent à la mondialisation ou qu'elles la forgent de manière proactive, la question se pose de savoir comment elles coordonnent des activités globalisées et s'il ressort de cela, d'abord, un modèle unique de structure des firmes globales ou plusieurs, ensuite, des formes de structures nouvelles ou non par rapport à ce qu'on connaissait jusque-là (cf. les trois derniers articles de ce numéro). ${ }^{5}$

Mais avant d'entrer dans les articulations entre mondialisation et transformation des entreprises, il faut revenir sur la mondialisation elle-même. Sur la longue durée, distinguons trois temps de la mondialisation. L'articulation entre mondialisation et transformation des entreprises doit en effet toujours être resituée dans l'un ou l'autre de ces temps.

5 Remercions ici chaleureusement les reviewers anonymes des articles ainsi que nos collègues d'Entreprises et Histoire: Patrick Fridenson, Arielle Haakenstad, Sylvain Lenfle et Nadège Sougy pour leur aide précieuse dans la préparation de ce numéro. 


\section{I - LES TROIS MONDIALISATIONS : PROTO-MONDIALISATION, MONDIALISATION INDUSTRIELLE, GLOBALISATION}

Les analyses insistent bien souvent sur le caractère inédit de la globalisation. L'historien Adam McKeown s'est déjà interrogé sur ce «processus continuellement obsédé par sa propre nouveauté » ${ }^{6}$. Cette obsession fait courir le risque d'une forme de présentisme qui invite à replacer ces phénomènes dans le temps long pour mieux en faire ressortir la singularité - et non pas nécessairement la nouveauté 7 . Ce détour par l'histoire pose cependant toute une série de difficultés méthodologiques. Comme le souligne Sebastian Conrad, l'histoire de la globalisation n'épuise pas toute l'approche d'histoire globale qui s'est développée depuis plus d'une vingtaine d'années8. L'histoire de la globalisation n'est donc sans doute pas appréhendée de la même manière en fonction des disciplines voire des sub-disciplines. Même au sein de chacune d'elles, comme la Business History par exemple, on peut noter une pluralité d'approches9. Pour autant, on peut s'accorder sur l'existence de deux moments essentiels constitués par le développement d'une proto-mondialisation entre les $\mathrm{XV}^{\mathrm{e}}$ et XVIII ${ }^{\mathrm{e}}$ siècles, d'une part, et par celui d'une mondialisation liée au développement du capitalisme industriel qui parcourt le $\mathrm{XIX}^{\mathrm{e}}$ siècle pour se cristalliser à la fin de ce dernier. Ces deux processus peuvent être comparés à la globalisation actuelle qui s'affirme depuis les années 1980 et qui n'est pas non plus une période monolithique.

\section{La proto-mondialisation des économies-mondes ( $\mathrm{XV}^{\mathrm{e}}$-XVIII ${ }^{\mathrm{e}}$ siècles)}

L'histoire de la globalisation doit beaucoup à l'histoire du capitalisme et aux analyses en termes d'économies-monde développées par Fernand Braudel puis par Immanuel Wallerstein. Comme le rappelait Braudel, il faut toutefois distinguer les économies-monde de l'économie mondiale ${ }^{10}$. Alors

6 Adam McKeown, «Periodizing Globalization», History Workshop Journal, $\mathrm{n}^{\circ}$ 63, printemps 2007, p. 218-230, ici p. 219.

7 Sebastian Conrad, What is global history?, Princeton, Princeton University Press, 2016, p. 97 ; Richard Baldwin, The Great Convergence. Information Technology and the New Globalization, Harvard University Press, 2016.

8 Ibid., p. 98-101. Sur l'histoire globale: Alessandro Stanziani, Les entrelacements du monde: histoire globale, pensée globale: XVI $-X X I^{e}$ siècles, Paris, CNRS éditions, 2018 ; Chloé Maurel, Manuel d'histoire globale: Comprendre le "global turn» des sciences humaines, Paris, Armand Colin, 2014 ; Le Débat, «Écrire l'histoire du monde », $\mathrm{n}^{\circ}$ 154, mars-avril 2009 ; Revue d'histoire moderne et contemporaine, « Histoire globale, histoires connectées », $\mathrm{n}^{\circ}$ 54-4 bis, 2007.

9 Teresa da Silva Lopes, Christina Lubinski et Heidi Tworek (dir.), The Routledge companion to the makers of global business, New York, Routledge, 2019 ; Geoffrey Jones, « Globalization » in Geoffrey Jones et Jonathan Zeitlin (eds), The Oxford Handbook of Business History, Oxford, Oxford University Press, 2008, p. 141-170.

10 Fernand Braudel, Civilisation matérielle, économie et capitalisme, tome 3, Le temps du monde, Paris, A. Colin, 1979, p. 12. 
que «l'économie mondiale s'étend à la terre entière » les économies-mondes s'étendent sur un espace géographique donné autour d'un centre urbain et s'organisent concentriquement autour de lui ${ }^{11}$. Même si les économies-monde sont centrées sur elles-mêmes, elles ne sont pas tout à fait autarciques. La circumnavigation entreprise par Fernand de Magellan et achevée par Jean Sébastien Elcano entre 1519 et 1522 , l'expansion espagnole et portugaise puis la mondialisation ibérique marquent des débuts d'une proto-mondialisation qui voit se développer les échanges entre ces économies-monde ${ }^{12}$. Elle s'étend jusqu'à la guerre de Sept Ans quand la France et la Grande-Bretagne s'opposent à l'échelle du monde au milieu du XVIII ${ }^{\mathrm{e}}$ siècle. Si cette période est souvent analysée comme un moment de l'expansion européenne, il importe de ne pas oublier la force de ces économies-monde extra-européennes. Au milieu du XVIII ${ }^{\mathrm{e}}$ siècle la Chine et le sub-continent indien produisent près de la moitié des biens manufacturés dans le monde et notamment des produits de consommation comme les indiennes, importées en Europe avant que ne s’y développe une industrie locale13.

La proto-mondialisation possède des particularités fortes. La masse des échanges à l'échelle du monde reste très faible par rapport à l'ensemble de la production, même si ces échanges peuvent être décisifs sur le plan quantitatif. De nouvelles formes d'entreprise apparaissent - que l'on songe aux différentes Compagnies des Indes orientales qui se dotent de nouvelles structures actionnariales et qui sont adossées à des monopoles accordés par le pouvoir politique. Cette proto-mondialisation repose aussi sur des migrations importantes, parfois forcées comme en témoigne les plus de huit millions d'esclaves africains soumis à la traite transatlantique du $\mathrm{XVI}^{\mathrm{e}}$ à la fin du XVIII $\mathrm{X}^{\mathrm{e}}$ siècle. Si les différences sont nettes entre cette proto-mondialisation et la globalisation actuelle - les ressources naturelles ne sont guère considérées comme des «biens communs mondiaux »-, il existe des similitudes qu'il s'agisse du caractère régional des échanges ou du caractère multipolaire du systèmemonde. À côté de l'Europe, d'autres économies-monde tout aussi puissantes existent alors, technologiquement aussi développées et aux niveaux de vie moyens comparables : la Chine, l'Inde, l'Insulinde, la Russie et l'économie-monde moyen-orientale.

11 Fernand Braudel, La dynamique du capitalisme, Paris, Flammarion, 2014 (1 ${ }^{\text {ere }}$ édition en 1985 chez Arthaud), p.76-80.

12 Le terme de «proto-mondialisation » est utilisé l'historien François Crouzet en 2003 pour évoquer les échanges entre économies-monde : Dominique Barjot et al., «La mondialisation », Entreprises et Histoire, $\mathrm{n}^{\circ} 32$, 2003, p.140). Son pendant anglais de «proto-globalization» est employé de manière plus systématique dans l'ouvrage dirigé par l'historien britannique Anthony Hopkins : Anthony G. Hopkins, Globalization in World History, New York, Norton \& Co., 2002.

13 Paul Bairoch, «International industrialization levels from 1750 to $1980 »$, Journal of European Economic History, $\mathrm{n}^{\circ} 11,1982$, p. 269-333; Patrick Verley, L'échelle du monde. Essai sur l'industrialisation de l'Occident, Paris, Gallimard, 1997, p. 161-179. 


\section{La mondialisation au temps du capitalisme industriel}

Le mouvement de mondialisation qui se développe au XIX ${ }^{\mathrm{e}}$ siècle avec l'essor du capitalisme industriel en Europe et aux États-Unis est souvent identifié comme l'un des jalons de la globalisation actuelle. Il se cristallise au tournant des $\mathrm{XIX}^{\mathrm{e}}$ et $\mathrm{XX}^{\mathrm{e}}$ siècles au cours de la période qui débute avec la Grande Dépression des années 1870 et qui s'achève à la veille de la Première Guerre mondiale. Ses caractéristiques sont bien connues. Ce temps de la mondialisation est encouragé par le changement de la structure productive en Europe et aux États-Unis au profit de l'industrie et par l'essor du commerce international qui crô̂t deux fois plus vite que la production. Cette ouverture commence dans la première moitié du $\mathrm{XIX}^{\mathrm{e}}$ siècle, grâce à la baisse du coût des transports et aux mesures adoptées en faveur du libre-échange - dont se gardent cependant les États allemands et les ÉtatsUnis14. Cette mondialisation se fait cependant aux dépens des industries non-occidentales. En Inde, par exemple, l'industrie textile ploie sous le poids de la concurrence des biens manufacturés britanniques et de la pression coloniale. En outre, les importantes vagues de migrations qui touchent l'espace transatlantique - environ 60 millions d'Européens émigrent en Amérique durant cette période - jouent un rôle important en encourageant une convergence des salaires et même des économies15. Par ailleurs, le rôle de la mobilité des capitaux, encouragé par l'adoption progressive du Gold Standard, est aussi un trait de cette mondialisation : au XIX ${ }^{\mathrm{e}}$ siècle, le taux de croissance annuel moyen du stock des investissements brut réalisés en dehors de leurs pays d'origine dépasse les $4 \%$. Enfin, un certain nombre de régulations internationales sont mises en place qu'il s'agisse de l'Union postale universelle (1874) ou de l'Union internationale pour la protection de la propriété industrielle (1883). À cet égard, le développement de la propriété intellectuelle va permettre à ces grandes entreprises de bénéficier de monopoles reposant sur une base technologique.

Il peut paraître paradoxal que la fin du $\mathrm{XIX}^{\mathrm{e}}$ siècle et le début du $\mathrm{XX}^{\mathrm{e}}$ siècle aient pu être considérés comme le cœur d'une «première mondialisation »16. La vague de mesures protectionnistes lancée à

14 Kevin H. O’Rourke et Jeffrey G. Williamson, «When did globalisation begin? », European Review of Economic History, vol. 6, $\mathrm{n}^{\mathrm{o}}$ 1, 2002, p. 23-50; Michel Fouquin, Jules Hugot et Sébastien Jean, « II. Une brève histoire des mondialisations commerciales », in CEPII, L'économie mondiale 2017, Paris, La Découverte, 2016, p. 22-38 ; Giovanni Federico et Antonio Tena-Junguito, « A tale of two globalizations: gains from trade and openness 1800-2010», Review of World Economics, vol. 153, n 3, 2017, p. 601-626.

15 Kevin H. O’Rourke et Jeffrey G. Williamson, Globalization and History. The Evolution of a NineteenthCentury Atlantic Economy, Cambridge (Mass), MIT Press, 1999, chapitre 8 et 10 ; Kevin H. O'RouRKE, «The era of free migration: Lessons for today », in Andrew Sobel (dir.), Challenges of Globalization: Immigration, Social Welfare, Global Governance, New-York, Routledge, 2009, p. 58-77.

16 Suzanne Berger, Notre première mondialisation : leçons d'un échec oublié, Paris, Seuil, 2003. 
la faveur de la Grande Dépression met fin à la dynamique d'ouverture qui avait caractérisé la première moitié du XIX ${ }^{\mathrm{e}}$ siècle sans pour autant en annuler les effets 17. Mais comme le souligne Patrick Verley dans son débat avec Jean-Philippe Robé dans ce numéro, la contradiction n'est qu'apparente. Les barrières douanières qui se dressent dans les années 1890 sont contournées par les entreprises multinationales qui se développent dans le monde. En fait, l'expansion des multinationales va de pair avec celle des infrastructures de transport et de communication, même si, dès cette époque, les multinationales jouent aussi un rôle essentiel dans le secteur minier et agricole18. Alors que cette période est celle qui voit l'émergence de «l'entreprise industrielle moderne » au sens chandlérien du terme, Mira Wilkins observe au même moment la cristallisation d'un «modèle américain » de firmes multinationales implantant des sites de production en dehors de leurs pays d'origine19. Ces acteurs vont jouer un rôle fondamental dans la mondialisation au $\mathrm{XX}^{\mathrm{e}}$ siècle et plus particulièrement dans celle qui s'opère à la fin de ce dernier.

\section{«La » mondialisation actuelle (1980-aujourd'hui) et ses inflexions}

La mondialisation que nous vivons aujourd'hui, dont l'on date souvent l'essor du tournant des années 1970 et 1980, est issue elle aussi d'un long mouvement de libéralisation des échanges, entamé longtemps auparavant, d'abord dans le cadre des négociations du GATT à partir de 1948. Les Trente Glorieuses constituent une période de relative clôture des économies ou du moins d'ouverture contrôlée, progressive, dans une logique de rattrapage de l'Europe et du Japon vis-à-vis des ÉtatsUnis. Avec la sortie du système de Bretton Woods par Nixon en 1971 et les politiques de libéralisation entamées à partir de 1980, relayées ensuite à travers une série d'accords de commerce multilatéraux, commence une période d'ouverture commerciale sensiblement plus significative, qui renoue avec les taux d'ouverture des économies qu'on observait durant la première mondialisation. La création de

17 Michel Fouquin, Jules Hugot et Sébastien Jean, « Une brève histoire des mondialisations commerciales », op. cit., p. 25.

18 Mira Wilkins, «Multinational Enterprise to 1930. Discontinuities and Continuities » in Alfred D. Chandler et Bruce Mazlish (eds), Leviathans. Multinational Corporations and the New Global Economy, Cambridge, Cambridge University Press, 2005, p. 45-79, ici p. 51-67.

19 Le caractère américain de ce modèle doit beaucoup au terrain d'analyse des premiers travaux de Mira Wilkins et notamment de son ouvrage The Emergence of Multinational Enterprise: American Business Abroad Colonial Era, Cambridge (Mass.), Harvard University Press, 1970 suivi de The Maturing of Multinational Enterprise : American Business Abroad from 1914 to 1979, Cambridge (Mass.), 1974 ; Mira WILKINS, The History of Foreign Investment in the United States to 1914, Cambridge (Mass.), Harvard University Press, 1989 : Id., The History of Foreign Investment in the United States, 1914-1945, Cambridge (Mass.), Harvard University Press, 2004. 
l'Organisation Mondiale du Commerce (OMC) en 1995 signe une seconde phase de cette ouverture commerciale conçue par certains comme une phase de $«$ reglobalisation $»^{20}$.

Cette mondialisation n'est pas un bloc. Pour faire simple, on peut distinguer deux sous-périodes de la mondialisation actuelle dont le tournant serait symbolisé par la création du G20 en 1999 et l'adhésion de la Chine à l'OMC en 2001. Les traits distinctifs de chacun de ces moments sont la variation d'ampleur des échanges commerciaux mondiaux ainsi que la nature de leurs acteurs et de leur hiérarchie. S'y ajoutent l'expansion, la vitesse et l'instabilité des marchés financiers ainsi que l'émergence d'une gouvernance mondiale, pour l'environnement et le climat notamment ${ }^{21}$. «La » mondialisation actuelle est donc loin d'être mouvement monolithique, continu, c'est un phénomène profondément dynamique : travailler et vivre dans la mondialisation de 1979, dans celle de 1999 ou dans celle de 2019 est très différent.

Cette distinction permet d'enrichir la comparaison entre la globalisation de la fin du XIX $\mathrm{X}^{\mathrm{e}}$ siècle et celle de la fin du $\mathrm{XX}^{\mathrm{e}}$ siècle, sujet de débats récurrents ${ }^{22}$. Il est bien établi que les taux d'ouverture commerciale à la veille de la Première Guerre mondiale sont similaires à ceux des débuts de la vague actuelle de mondialisation. Des différences significatives existent cependant comme celle qui concerne les zones géographiques impliquées. À côté de la Triade et de régions qui rappellent les « économies-monde » de la proto-industrialisation - Chine, Inde, Russie, Indonésie (qui recouvre en bonne partie l'ancienne Insulinde), Arabie Saoudite (qui ne recouvre qu'une partie de l'ancienne économie-monde du Moyen-Orient) - des puissances économiques comme le Brésil ou l'Afrique du Sud s'affirment. En outre, la mondialisation du $\mathrm{XXI}^{\mathrm{e}}$ siècle voit s'accentuer les interdépendances entre ces ensembles qui touchent désormais l'ensemble de la planète.

La mondialisation actuelle se distingue fortement des deux précédentes vagues sur trois plans. La question de la nature comme bien commun mondial et comme enjeu d'action concertée à l'échelle du monde est posée. Ce problème du climat et de la biodiversité n'est rien moins que cardinal pour l'avenir de l'humanité et il pose la question de l'invention d'une nouvelle gouvernance mondiale. La globalisation n'est pas l'internationalisation, c'est même une forme d'affaiblissement de

20 Ronald Findlay et Kevin O'Rourke, Power and Plenty: Trade, War, and the World Economy in the Second Millennium. Princeton, Princeton University Press, 2007.

21 Cécile Bastidon Gilles et al., Histoire de la globalisation financière: Essor, crises et perspectives des marchés financiers internationaux, Armand Colin, 2010.

22 Michel Fouquin, Jules Hugot et Sébastien Jean, « Une brève histoire des mondialisations commerciales », op. cit., Richard E Baldwin et Philippe Martin, «Two Waves of Globalisation: Superficial Similarities, Fundamental Differences », Note de recherche du National Bureau of Economic Research, $n^{\circ}$ 6904, 1999 ; Gérard Kébadjian, «Analyse économique et mondialisation: six débats », in M. Beaud et al. (dir.), Mondialisation. Les mots et les choses, op. cit., p.51-77, en particulier, p.53-56. 
l'internationalisation. C'est le deuxième point : les États-nations qui s'étaient développés lors des mondialisations précédentes voient leur rôle remis en question - voire affaibli - par la globalisation actuelle. Enfin, les entreprises sont appelées à participer à cette gouvernance nouvelle pour résoudre certains des problèmes globaux, à commencer par celui de leurs externalités négatives au regard du climat et de la biodiversité. En rapport aux trois temps de la mondialisation et à ses formes multiples (mondialisation du capital, du travail, de la nature ou des flux de biens et services), ce sont ces entreprises et leurs transformations qui sont au cœur de ce numéro.

\section{II - LES TRANSFORMATIONS DES ENTREPRISES MULTINATIONALES : CONSEQUENCE DE LA GLOBALISATION OU MOTEUR DE CETTE GLOBALISATION?}

$\mathrm{Si}$ « la »globalisation est une notion commode, la question qui doit être posée est celle des rapports qu'entretient la globalisation des entreprises avec les autres dimensions de globalisation. Tout d'abord, la croissance des échanges commerciaux mondiaux ne peut pas être pensée indépendamment de l'évolution des activités internes aux entreprises. Au cours de la globalisation actuelle, on estime qu'entre le tiers et un peu moins de la moitié de ce qu'on mesure à travers les statistiques du « commerce mondial » correspond en fait à des transferts intra-entreprises, destinés à faire passer les composants des biens et services d'une étape de leur réalisation à une autre au sein des différentes implantations de la firme ${ }^{23}$. La croissance du commerce global est ainsi due, pour la moitié du phénomène environ, à la globalisation interne des entreprises, c'est-à-dire à la répartition de leurs unités opérationnelles pensée à l'échelle globale.

Ensuite, la mondialisation financière, qui se caractérise notamment par de nouvelles formes d'engagement de l'actionnariat, joue un rôle déterminant dans la structuration des performances que doivent atteindre prioritairement les entreprises cotées et elle influe, par conséquent, sur les choix d'implantation géographique et d'externalisation d'activités ${ }^{24}$. Cela a un effet en retour tant sur la mise en compétition généralisée des territoires par les multinationales - un point essentiel souligné par l'économiste de la mondialisation Pierre-Noël Giraud - que sur la tentation pour elles de s'exonérer de la prise en charge de leurs externalités et des atteintes aux biens publics mondiaux -

23 Jean Piel, «De quelques considérations lexicales et historiques à propos de la 'mondialisation' », op.cit, p.166; Charles-Albert Michalet, 2005, p.37.

24 Tristan Auvray et alii, L'entreprise liquidée. La finance contre l'investissement, Paris, Michalon, 2016 ; Rachelle Belinga, L'engagement actionnarial : de l'industrialisation de l'actionnariat à l'expression de nouvelles responsabilités, thèse en sciences de gestion, Mines ParisTech - PSL Research University, 2018. 
donc sur la globalisation de la nature ${ }^{25}$. Cette compétition touche aussi la main d'œuvre. Le caractère structurant de la distinction entre les emplois directement soumis à la compétition internationale («emplois nomades ») et ceux qui en sont protégés («emplois sédentaires ») dans certaines analyses économiques de la mondialisation actuelle est une autre expression du fait que, malgré de faibles flux de main d'œuvre, on peut parler de mondialisation du travail - la mondialisation des entreprises jouant sur les emplois nomades qui jouent à leur tour sur les emplois sédentaires ${ }^{26}$.

Par conséquent, aucun aspect de la mondialisation actuelle ne peut être séparé de la globalisation des entreprises ! L'importance prise par ces dernières est telle qu'il devient impossible de raisonner sur la régulation ou la gouvernance mondiale sans prendre en compte leur place au sujet de laquelle les réflexions ne cessent de s'intensifier ${ }^{27}$. La question du droit et des rapports de pouvoirs entre acteurs, notamment entre entreprises, Etats et ONG, prend un relief tout particulier28. Dans ce contexte, il devient très important d'essayer de démêler les fils, de bien distinguer les différents liens entre les transformations des entreprises aux globalisations en cours. Tel est l'objet de ce numéro d'Entreprises et histoire, dont plusieurs contributions sont issues d'un colloque tenu en 2014 à l'Université Bocconi $^{29}$. S'il rappelle des facteurs essentiels de la globalisation actuelle, il met aussi en lumière combien la réinvention des entreprises dans ce contexte non seulement prend des formes diverses mais encore repose sur une organisation de cette diversité. En outre, il souligne la multiplicité des temporalités à l'œuvre montrant que ces processus de transformation consistent à se donner du temps voire à s'inscrire dans une histoire séculaire - ce qui montre bien que la réflexion sur les rapports entre les différentes vagues de mondialisation est tout sauf accessoire. Trois faisceaux d'interrogations structurent ce numéro. Le premier porte sur certains des facteurs de globalisation des entreprises (comment d'autres volets de la globalisation pèsent sur la globalisation des entreprises) et le deuxième sur la contribution active des entreprises à la globalisation. Enfin, le dernier concerne la

25 Pierre-Noël Giraud, Les Globalisations. Émergences et fragmentations, éditions Sciences Humaines, 2018, p. 38. Sur le même thème, voir aussi Pierre-Noël Giraud, L'inégalité du monde, Paris, Gallimard, 1996.

26 Pierre-Noël Giraud, Les Globalisations..., op. cit., p. 78.

27 Olivier Basso, Politique de la très grande entreprise: leadership et démocratie planétaire, Paris, Presses universitaires de France, 2015 ; «Les stratégies politiques des organisations », Revue française de gestion, $\mathrm{n}^{\circ}$ spécial, 7, 2015.

28 Jean-Philippe Robé, «Les États, les entreprises et le droit. Repenser le système-monde », Le Débat, $\mathrm{n}^{\circ} 161$, septembre-octobre 2010, p.74-87; Id., «Les entreprises multinationales, vecteurs d'un nouveau constitutionnalisme » in Jean-Philippe Robé, Le temps du monde de l'entreprise, Paris, Dalloz, 2015, p. $570-594$.

29 Le colloque «Capitalism and the Corporation: Today and Yesterday » s'est tenu du 23 au 25 octobre 2014 à l’Université Bocconi à l'initiative de Francesco Amatori et de Luigi Orsenego. 
coordination par les entreprises de leurs activités globalisées et par l'émergence - ou non - de nouvelles structures ou modes de coordination.

\section{- Les facteurs de globalisation des entreprises}

Il était nécessaire de revenir d'abord sur les facteurs exogènes de l'actuelle mondialisation exogènes du point de vue des entreprises. L'article d'Antoine Frémont traite du rôle fondamental du transport maritime et de la conteneurisation dans l'expansion du commerce international dès les années 1950 qui a pour effet de pousser les entreprises à délocaliser leurs activités voire à les externaliser. Il analyse les effets en retour de la mondialisation sur l'industrie du transport maritime elle-même, qui doit faire face à des questions sociales en environnementales accrues par l'intensification du trafic.

Tristan Auvray et Thomas Dallery reviennent, quant à eux, sur une autre dimension essentielle de la globalisation: la dimension financière et plus particulièrement la relation entre actionnaires et entreprises, qu'ils analysent à travers les droits de vote des actionnaires aux assemblées générales et la liquidité des actions. Ils montrent ainsi tout à la fois continuité et différence d'une mondialisation à l'autre. En exceptant le cas de l'actionnariat de soutien, engagé à moyen-long terme auprès des entreprises et toujours présent d'une mondialisation à l'autre, ils montrent qu'aussi bien pendant la première mondialisation financière que pendant la seconde, la liquidité des capitaux tend à opposer finance et investissement. La différence d'une mondialisation à l'autre tient à l'hyper-liquidité des capitaux dans la phase actuelle, dont ils montrent l'importance et les conséquences sur la conduite des entreprises.

Enfin, prenant à rebours la position de Thomas Levitt selon laquelle la globalisation se traduirait par une homogénéisation forcée des marchés, les entreprises visant des économies d'échelle aussi grandes que possible, l'article d'Emanuela Scarpellini montre comment, malgré certaines convergences des goûts, s'opère une forte différentiation des marchés nationaux, notamment du fait de dynamiques sociales. Ces singularités des marchés nationaux ou locaux, reconnues par les entreprises et acceptées par elles, ont alors des répercussions sur les logiques de production - des productions flexibles et non pas de masse.

Dans ces articles, on le voit, les entreprises subissent la mondialisation financière, s'adaptent à la mondialisation partiellement divergente des marchés des biens de consommation ou saisissent les opportunités de la mondialisation permises par l'évolution des transports. À côté de cette logique 
adaptative, il existe une logique plus pro-active par laquelle les entreprises font aussi la mondialisation, la forgent, lui donnent son visage particulier. C'est cette part motrice des entreprises dans la mondialisation qu'examine la seconde série de trois articles dans ce numéro.

\section{- Les entreprises, dépendantes de la globalisation ou motrices de cette globalisation ?}

Cet ensemble de contributions porte sur la transformation des activités des entreprises et plus précisément - dans l'ordre des articles - sur la production, sur la conception des produits, enfin sur la redéfinition même de ce qu'est l'activité de l'entreprise. Plusieurs échelles d'analyse sont mobilisées.

David Koistinen et Kenneth Lipartito s’intéressent ainsi à la répartition géographique de la production dans le secteur de l'électronique. Pour les auteurs, un mouvement de la délocalisation de la production entamé dès les années 1950 a préparé l'émergence de réseaux globaux de production du milieu des

années 1980. Sans reprendre les termes précis du débat ouvert par Paul Krugman sur la responsabilité de la mondialisation dans le chômage industriel des pays développés, les auteurs le prolongent et le renouvellent à leur manière. Ils font écho aux analyses de Garry Pisano et Willy Shih qui montrent ses effets destructeurs pour les États-Unis, en termes de compétences des entreprises d'abord, de compétitivité et d'emploi industriel ensuite, des politiques d'externalisation et de délocalisation de la production. $^{30}$

Les processus d'innovation ne sont pas à l'écart du mouvement de globalisation ${ }^{31}$. L'article de Cédric Dalmasso s'attache à une forme particulière touchant une activité de conception - le développement. À partir de l'étude de l'ouverture d'un centre de recherche-développement d'un grand constructeur automobile français, il montre que l'échec ou le succès d'une telle externalisation suppose la bonne prise en compte des compétences dans le pays d'accueil. Or ces dernières sont tributaires d'un ensemble de paramètres - le système éducatif ou le marché de l'emploi, par exemple - qui ne sont pas toujours bien anticipés. Même dans le domaine de la conception, la globalisation demande du temps.

30 Garry P. Pisano et Willy C. Shih, « Restoring american competitiveness », Harvard Business Review, vol. 87, $\mathrm{n}^{\circ}$ 7/8, p. 114-125; Id., Producing prosperity: Why America needs a manufacturing renaissance, Cambridge (Mass.), Harvard Business Press, 2012.

31 Sihem Ben Mahmoud-Jouini et alii (dir.), «Globalisation des processus d'innovation dans les entreprises multinationales», numéro spécial, Management international, vol. 19, n4, été 2015. 
Cette question de la temporalité est reprise par James W. Cortada sur une chronologie plus large. Il s’interroge sur la longévité d'IBM, une entreprise maintenant séculaire qui se conçoit d'emblée comme internationale, en montrant comment elle parvient à se réinventer en redéfinissant la nature même de ses activités, en faisant les investissements nécessaires et en créant une culture spécifique. Il offre par la même occasion une analyse des traits caractéristiques de ce que l'on pourrait appeler des entreprises de longue durée («long-lived firms » dans son texte).

\section{- Structure et coordination des multinationales à l'ère de la globalisation : du nouveau ?}

La dernière partie du numéro porte sur l'évolution de la structure des entreprises dans la mondialisation. Elle fait une grande place en effet à ce que l'on pourrait appeler l'actualité du chandlerisme confrontée à cette nouvelle vague de mondialisation ${ }^{32}$. Mais comme le rappelle $\mathrm{W}$. Mark Fruin, le cadre d'analyse chandlerien n'est pas figé et se caractérise par une certaine plasticité dont témoignent les articles qui suivent ${ }^{33}$. Dès 2006 Suzanne Berger mettait en garde contre toute nouvelle tentation de « one best way » à l'ère, pourtant, de l'hyper-compétition globale et du « made in monde $»^{34}$. Confortant cette analyse attentive tant à l'histoire qu'à la variété des modèles possibles, les trois articles de la dernière partie de ce numéro illustrent la forte diversité des structures d'entreprises à l'ère de la globalisation.

Dans leur article, Asli M. Colpan et Takashi Hikino analysent le rôle des sièges sociaux dans la longue durée du capitalisme industriel et son évolution récente. La transformation de la firme $\mathrm{M}$ et l'émergence de nouvelles formes organisationnelles dans un contexte de globalisation - et notamment les groupes - conduisent à redéfinir la place des sièges sociaux ainsi que le rapport entre actionnaires et managers. À cet égard, cet article fait aussi bien écho à la contribution de Tristan Auvray et de Thomas Dallery qu'à celui de James Cortada en posant la question du lieu de ce rapport de force qui devrait aussi se poser pour celui qui rassemble managers et représentants des salariés. Les transformations de la structure des entreprises ne sont cependant pas sui generis.

32 Naomi R. Lamoreaux, Daniel M. G. Raff et Peter Temin, «Beyond Markets and Hierarchies: Toward a New Synthesis of American Business History », The American Historical Review, vol. 108, no 2, 2003, p. 404-433 ; Thomas K. McCraw, «Alfred Chandler: His Vision and Achievement », Business History Review, vol. 82, $\mathrm{n}^{\circ} 02,2008$, p. 207-226.

33 W. Mark Fruin, « Globalization and Alfred D. Chandler's modern (American) firm: an essay », Journal of Management History, vol. 15, $\mathrm{n}^{\circ}$ 3, 2009, p. 261-271

34 Suzanne Berger, Made in monde : les nouvelles frontières de l'économie mondiale, Paris, Le Seuil, 2006. 
L'étude du secteur du vin de Porto après la Seconde Guerre mondiale menée par Teresa Da Silva Lopes illustre l'importance du secteur de l'agro-alimentaire dans le processus de globalisation. Elle montre que les coûts de transaction au sein de ce marché ont conduit à privilégier une intégration horizontale alors que d'autres secteurs comme celui du champagne optait pour une intégration horizontale et verticale. Le résultat de cette évolution tout à fait particulière aboutit à la survie de marques très anciennes alors même que les firmes qui les avaient créées ont disparu. Preuve que la globalisation est loin de faire table rase du passé et qu'au-delà de la structure, la pérennité du capital - fût-il immatériel - reste essentielle.

Enfin, W. Mark Fruin et Simon A. Rodan prolongent et renouvellent la typologie que Chandler avait construite dans Scale and Scope pour les firmes du capitalisme industriel. Les deux auteurs nous incitent à voir au-delà de la firme unique et construisent un modèle multi-firmes où la firme-réseau tend à devenir centrale. Dans ce contexte de concurrence renforcées, cette nouvelle structuration va de pair avec de nouvelles formes de management et invite à repenser la définition même de l'entreprise.

Outre ces contributions, ce numéro comporte ses traditionnelles rubriques en lien avec le thème général. Dans un entretien croisé, on l'a vu, Jean-Philippe Robé et Patrick Verley reviennent sur les temps et les enjeux de la mondialisation. Le document, présenté par Patrick Fridenson, permet d'appréhender l'implantation de Renault en Iran grâce au témoignage d'un de ses principaux acteurs : Louis Schweitzer. Au-delà des spécificités de l'Iran, ce document permet d'appréhender des tendances générales concernant les rapports entre les multinationales de l'automobile et les pays émergents. La rubrique « Nouvelles des archives » nous rappelle que la globalisation concerne aussi l'activité de documentation et d'archivage des entreprises multinationales. Niels Viggo Haueter nous montre que les archives de la compagnie de réassurance Swiss Re, fondée en 1863, ont été très tôt confrontées à ces questions posées à l'échelle du monde. Enfin, le clin d'œil de Phil Scranton évoque la confiance que l'on peut apporter à l'information produite par ses partenaires commerciaux quand ils appartiennent au camp d'en face. C'est poser là la question de l'intégration des pays communistes dans la mondialisation et celle des préjugés que cette dernière peut parfois susciter.

L'ensemble des articles de ce numéro spécial montre bien la complexité des processus à l'œuvre dans les entreprises de cette ère globale. Ils permettent cependant d'aller au-delà en identifiant des enjeux cruciaux. Le premier porte sur la nature de la transformation qui caractérise la globalisation. Là, il ne s'agit pas de la redéfinition du concept mais bien de la transformation des choses qui sont impliquées 
dans ce mouvement. La globalisation se caractérise certes par une accélération des échanges et une intensification des interdépendances. Mais cette dynamique change les produits, les activités et peutêtre mêmes les êtres eux-mêmes. C'est là faire écho à une analyse déjà suggérée par McLuhan. L'autre enjeu porte sur les temporalités. Il est tout à fait frappant que bon nombre des études produites se placent dans un temps relativement long, remontant bien souvent au temps du capitalisme industriel. Cela paraît nécessaire, bien sûr, pour mieux comprendre la singularité de la globalisation actuelle. Mais, au-delà de cet aspect méthodologique classique, plusieurs articles suggèrent bien qu'une certain prise de distance temporelle est nécessaire pour les entreprises et leurs acteurs. Au sentiment d'immédiateté inspiré par les moyens de communication doit répondre une maîtrise du temps pour construire des compétences, déployer des projets ou faire vivre des marques séculaires. Il en va d'ailleurs de même pour toutes les formes d'idiosyncrasie qui font le quotidien de la globalisation. Contrairement à ce que pouvait penser Theodore Levitt, les firmes n'ont pas trouvé un intérêt à aplanir toute forme de singularité ; au contraire, les singularités peuvent s'avérer être un atout pourvu qu'elles soient prises en charge par une organisation adéquate ${ }^{35}$. Enfin, la globalisation n'est pas un processus linéaire et irréversible. Les effets de la crise financière de 2008, la résurgence du protectionnisme et certains phénomènes de relocalisation font naître une curiosité à la fois politique et scientifique pour la « dé-globalisation » alors même que se construisent les nouvelles routes de la soie.

35 Rawi Abdelal et Richard S. Tedlow, «Theodore Levitt's "The Globalization of Markets": An Evaluation after Two Decades », in John A. Quelch et Rohit Deshpande (dir.), The global market: developing a strategy to manage across borders, San Francisco, Jossey-Bass, 2004, p. 11-30. 